\title{
Sensitivity Analysis on Locations of Energy Storage in Power Systems With Wind Integration
}

\author{
Nhi T. A. Nguyen, Student Member, IEEE, Duong D. Le, Member, IEEE, Godfrey G. Moshi, Member, IEEE, \\ Cristian Bovo, Member, IEEE, and Alberto Berizzi, Member, IEEE
}

\begin{abstract}
The penetration of renewable energy sources, particularly wind energy, into power systems has been rapidly increasing in recent years. However, the integration of wind power has posed many challenges for power system operation. For instance, this type of energy source is relatively variable and unpredictable. The installation of this renewable source might require the grid to transmit power at full capacity and some transmission lines could become congested. As a result, in some operating conditions, wind power could be curtailed, which will drive up costs for system operators. One of the actions that can be taken to support the integration of the wind is using energy storage systems. In this paper, a multiperiod ac optimal power flow problem with battery energy storages (BESs) is formulated and sets of candidate buses for BES installation are identified based on an economic criterion. Tests are carried out on IEEE 14-bus and IEEE 118-bus systems to assess the robustness of storage location on system operation.
\end{abstract}

Index Terms-Curtailed wind, energy storage systems (ESSs), location, locational marginal price (LMPs), multiperiod, optimal power flow (OPF), production cost, sensitivity, wind integration.

\section{NOMENCLATURE}

$\begin{array}{ll}\eta_{\mathrm{ch}_{i}} & \text { Charging efficiency of the ESS at bus } i . \\ \eta_{d_{i}} & \text { Discharging efficiency of the ESS at bus } i . \\ \theta_{i}^{t} & \text { Voltage angle of bus } i \text { in hour } t . \\ \theta_{k}^{t} & \text { Voltage angle of bus } k \text { in hour } t . \\ B_{i}^{t} & \text { Energy of the ESS at bus } i \text { in hour } t . \\ B_{i}^{t-1} & \text { Energy of the ESS at bus } i \text { in hour } t-1 . \\ B_{i}^{\max } & \text { Upper limit of energy of the ESS at bus } i . \\ B_{i}^{\min } & \text { Lower limit of energy of the ESS at bus } i . \\ B_{i k} & \text { Line susceptance of branch } i k . \\ c_{0_{i}}, c_{1_{i}}, c_{2_{i}} & \text { Cost coefficients of generating units at bus } i . \\ c_{d_{j}}, c_{c h_{j}} & \text { Cost coefficients for charging and discharging } \\ G_{i k} & \text { power of the ESS at bus } j .\end{array}$

Manuscript received December 22, 2015; revised April 12, 2016; accepted June 14, 2016. Date of publication August 16, 2016; date of current version November 18, 2016. Paper 2015-IACC-0900.R1, presented at the 2015 IEEE 15th International Conference on Environment and Electrical Engineering (EEEIC), Rome, Italy, Jun. 10-13, and approved for publication in the IEEE TRANSACTIONS ON INDUSTRY APPLICATIONS by the Industrial Automation and Control Committee of the IEEE Industry Applications Society.

N. T. A. Nguyen, C. Bovo, and A. Berizzi are with the Politecnico di Milano, 20133 Milano, Italy (e-mail: thiai.nguyen@polimi.it; cristian.bovo@polimi.it; alberto.berizzi@polimi.it).

D. D. Le is with the Politecnico di Milano, 20133 Milano, Italy, and also with the Department of Electrical Engineering, Danang University of Science and Technology, Danang, Vietnam (e-mail: dinhduong.le@ polimi.it).

G. G. Moshi is with the Department of Electrical Engineering, Dar es Salaam Institute of Technology, Dar es Salaam, Tanzania (e-mail: godfreygladson.moshi@polimi.it).

Color versions of one or more of the figures in this paper are available online at http://ieeexplore.ieee.org.
$I_{i j}^{t}$

$I_{j i}^{t}$

$I_{i j}^{\max }$

$I_{j i}^{\max }$

$n$

$n b r$

$n g$

ns

$P_{i}^{t}$

$P_{\mathrm{ch}_{i}}^{\max }$

$P_{\mathrm{ch}_{i}}^{\min }$

$P_{c h_{i}}^{t}$

$P_{d_{i}}^{\max }$

$P_{d_{i}}^{\min }$

$P_{d_{i}}^{t}$

$P_{G_{i}}^{\max }$

$P_{G_{i}}^{\min }$

$P_{G i}^{t}$

$P_{L_{i}}^{t_{i}}$

$Q_{i}^{t^{i}}$

$Q_{c h i}^{\max }$

$Q_{\mathrm{ch}_{i}}^{\min }$

$Q_{\mathrm{ch}_{i}}^{t}$

$Q_{d_{i}}^{\max }$

$Q_{d_{i}}^{\min }$

$Q_{d_{i}}^{t}$

$Q_{G_{i}}^{\max }$

$Q_{G_{i}}^{\min }$

$Q_{G_{i}}^{t}$

$Q_{L_{i}}^{t}$

$T$

$V_{i}^{\max }$
Magnitude of the current flowing from bus $i$ to bus $j$ in hour $t$.

Magnitude of the current flowing from bus $j$ to bus $i$ in hour $t$.

Upper limit of the current flow from bus $i$ to bus $j$.

Upper limit of the current flow from bus $j$ to bus $i$.

Total number of buses.

Total number of branches.

Total number of generators.

Total number of ESSs installed.

Real power injection at bus $i$ in hour $t$.

Upper limit of real charging power of the ESS at bus $i$.

Lower limit of real charging power of the ESS at bus $i$.

Real charging power of the ESS at bus $i$ in hour $t$. Upper limit of real discharging power of the ESS at bus $i$.

Lower limit of real discharging power of the ESS at bus $i$.

Real discharging power of the ESS at bus $i$ in hour $t$.

Upper limit of real generation at bus $i$.

Lower limit of real generation at bus $i$.

Real generation power at bus $i$ in hour $t$.

Real power of load at bus $i$ in hour $t$.

Reactive power injection at bus $i$ in hour $t$.

Upper limit of reactive charging power of the ESS at bus $i$.

Lower limit of reactive charging power of the ESS at bus $i$.

Reactive charging power of the ESS at bus $i$ in hour $t$.

Upper limit of reactive discharging power of the ESS at bus $i$.

Lower limit of reactive discharging power of the ESS at bus $i$.

Reactive discharging power of the ESS at bus $i$ in hour $t$.

Upper limit of reactive generation at bus $i$.

Lower limit of reactive generation at bus $i$.

Reactive generation power at bus $i$ in hour $t$.

Reactive power of load at bus $i$ in hour $t$.

The optimization horizon.

Upper limit of voltage magnitude of bus $i$. 
Lower limit of voltage magnitude of bus $i$.

Voltage magnitude of bus $i$ in hour $t$.

Voltage magnitude of bus $k$ in hour $t$.

\section{INTRODUCTION}

$\mathbf{E}$ NERGY storage systems (ESSs) can be an alternative to manage wind power variability, and hence, provide flexibility and reliability for power systems with high wind penetration level. This technology enables electricity to be stored at times of high wind and low demand, and then, to be released at low wind, high demand periods. This ability to store electricity of ESSs can efficiently compensate for the intermittent behavior of wind power and provide economically optimal operation for wind generation.

Potential applications of ESSs for grid-connected wind generation are thoroughly addressed in [1]. ESSs can be applied for mitigating wind power curtailment due to limited transmission capacity, which helps avoid any required transmission capacity upgrade. ESSs can serve to shape a portion of wind generation, i.e., shifting a portion of wind generation from high wind, off-peak load periods to low wind, peak load periods, for optimizing the overall economics. Other applications of ESSs is hedging wind forecast uncertainties, and hence, increasing wind generation revenues. An overview on different energy storage technologies and their uses with renewable energy is provided in [2]. Additional studies on other applications of ESSs such as frequency control and risk mitigation can be found in [3]-[7].

The combined operation of the ESS and wind generation has drawn special attention in many studies. In [8], the ESS is used in combination with wind generation for wind curtailment reduction and price arbitrage. That paper investigates the optimal scheduling of the ESS cooperating with wind farms and connected to a distribution network. Daneshi and Srivastava [9] presented a security-constrained unit commitment model with wind and battery energy storage (BES) and discusses the role of BES on locational pricing, economic, peak load shaving, and transmission congestion management using an eight-bus system case study. An increasing interest in optimal operation strategies of the ESS in electricity markets, where electricity price is an uncertainty, has been found in [10]-[12]. Akhavan-Hejazi and Mohsenian-Rad [10] presented a stochastic programming framework on optimal bidding of independent storage units in the day-ahead and hour-ahead energy and reserve markets, while Hu et al. [11] developed an optimal operation strategy for the ESS in hourly spot markets so that profits for the storage system are maximized. In [12], optimal energy exchange with electricity markets for energy storage in wind power plants has been considered taking into account transmission constraints.

Dealing with placement of the ESS in power grids, in [13], a methodology is proposed to allocate the ESS in a distribution system with high wind penetration. The ESS is optimally placed and sized to both accommodate wind energy and minimize generation costs. Song et al. [14] used a sensitivity analysis method to find optimal locations of ESSs for reducing transmission congestion. Oh [15] proposed an approach for deploying storage devices and discusses the feasibility and economic impact of using storage devices. However, the approach proposed is tested on a small system, using the simplified dc optimal power flow (OPF) approximation. Ghofrani et al. [16], [17] and Dvijotham and Chertkov [18] proposed approaches to determine optimal placement and sizes of ESSs using the dc OPF model and perform tests on the IEEE 24-bus system. Ghofrani et al. [16], [17] used a two-point estimate method to optimally place ESSs in a deregulated power system with wind integration and the optimization problem is solved for each single period during the considered optimization horizon. Dvijotham and Chertkov [18] incorporate operation of ESSs into the planning problem with optimal control schemes. The placement of ESSs in power grids with both conventional and wind generation is also studied in [19]. The authors use a semidefinite relaxation ac OPF model to solve the optimal placement problem and perform tests on the IEEE 14-bus system. In [20], a dc OPF framework is proposed for storage portfolio optimization, including storage size, technology, and location in transmission-constrained power networks. The framework is tested with the IEEE 14-bus system. Xiong and Singh [21] proposed an approach employing a dc power flow model for determining the optimal location and size of an ESS in a power system with uncertain wind generation. Case studies are conducted with the IEEE 96-bus system and Benders decomposition algorithm is applied to reduce the computational burden.

Proposed approaches for optimal placement of the ESS have been demonstrated with only small systems. For larger systems, especially real-size ones, computational burden is still an issue. Therefore, for the best planning of ESSs, it is necessary to preliminarily identify the most suitable area or the best candidate locations for installing the ESSs. This is carried out, in the methodology proposed in this paper, based on an ac OPF multiperiod model with BESs and wind integration for time shifting [22] and congestion relieving applications. The main contribution of this study is identifying the best candidate locations for storage allocation based on an economic criterion and assessing the influence of storage location and size on production cost, amount of wind to be curtailed and marginal prices. To this goal, additionally, in this paper, the full ac OPF model is developed to capture realistic physical power flows of the system better than the dc approach and the relaxed models used in [15], [16], and [18]-[20]. This approach is also much more accurate and reliable when issues such as congestion and voltage constraints are concerned. Moreover, in this paper, the multiperiod OPF model is employed, which takes into account time interdependence, i.e., the problem is solved simultaneously for all periods of the optimization horizon. In this way, the intertemporal constraints relevant to the storage are considered. The multiperiod model has been demonstrated in [23] and [24] to be a more physically suitable approach to study the operation of a storage device and to provide better operational schedule: In this paper, the proposed model is adopted, for example, to shift wind power over time and get reduction of wind curtailment in case of transmission congestions, thus allowing an efficient utilization of transmission capacity.

The remainder of this paper is organized as follows: In Section II, the methodology is described. In Section III, tests with mod- 
ified IEEE 14-bus and IEEE 118-bus systems are described and results are discussed. Finally, Section IV concludes this paper.

\section{Methodology}

In this section, the methodology to define the most suitable candidate locations for ESSs is described. The optimization is carried out on the total generation cost that represents transmission system operator's (TSO) point of view; in other words, the goal is to improve the system operation regardless of the revenues of the single companies operating wind farms. ESSs, therefore, can be installed at any bus by the (TSO) in order to maximize the efficiency and security of the overall system. Moreover, the total time horizon considered (which could be either one week or one month or a set of representative weeks of the year) is discretized and the hourly operation is optimized considering the presence of the ESSs; a multiperiod approach is necessary to properly model the intertemporal constraint characteristic of the ESSs. The multiperiod ac OPF described in Section II-A provides as byproduct the Lagrange multipliers, which are used in Section II-B to define the best candidate locations for ESSs.

\section{A. Multiperiod AC OPF Mathematical Formulation}

A multiperiod ac OPF model with ESS integration can be formulated as follows [22].

1) Objective function: The objective function to be minimized in this model is the total production cost $\sum_{t=1}^{T}\left(\mathrm{PC}^{t}\right)$, using as control variables ESS charging/ discharging power and generation output of all dispatchable generators in each period $t$

$$
\begin{aligned}
O F= & \operatorname{Min} \sum_{t=1}^{T} \sum_{i=1}^{n g}\left[c_{0_{i}}+c_{1_{i}} P_{G_{i}}^{t}+c_{2_{i}}\left(P_{G_{i}}^{t}\right)^{2}\right] \\
& +\sum_{t=1}^{T} \sum_{j=1}^{n s}\left(c_{d_{j}} P_{d_{j}}^{t}-c_{\mathrm{ch}_{j}} P_{\mathrm{ch}_{j}}^{t}\right) \\
= & \operatorname{Min} \sum_{t=1}^{T}\left(\mathrm{PC}^{t}\right) .
\end{aligned}
$$

In order to take into account the variability of load and wind, two possible approaches are available: The first is to run the model on a longer time frame, e.g., one year. However, the tractability of the resulting problem is subject to the size of the system. The second approach, which is typically used in power system planning, is to run the model on a weekly basis, considering a number of weekly scenarios representative of the most significant loading and wind conditions (high wind-high load, high windlow load, etc.), weighting the results based on the weekly energies. Therefore, the value of $T$ depends on the time horizon adopted in the planning problem. In particular, in this paper, the optimization problem is run on a daily basis, thus $T=24 \mathrm{~h}$.

In (1), the first term is the production cost of all generating units. The second term is introduced so that at the optimal solution, the ESS is not charged and discharged at the same time. Hence, $c_{\mathrm{ch}}$ and $c_{d}$ are fictitious charging and discharging costs applied to the ESS. When charging, the ESS is treated as a load with the fictitious charging cost set equal to zero $\left(c_{\mathrm{ch}}=0\right)$. To prevent simultaneous charging and discharging, the discharging cost $c_{d}$ is set to a very small quantity, e.g., $c_{d}=10^{-2}$, as presented in [25].

2) Equality constraints.

1) Power balance equations: Include equations for real and reactive power at each node $i$ in each time period $t$

$$
\begin{aligned}
& P_{i}^{t}=P_{G_{i}}^{t}-P_{L_{i}}^{t}+P_{d_{i}}^{t}-P_{\mathrm{ch}_{i}}^{t} \\
& =\sum_{i=1}^{n} V_{i}^{t} V_{k}^{t}\left[G_{i k} \cos \left(\theta_{i}^{t}-\theta_{k}^{t}\right)+B_{i k} \sin \left(\theta_{i}^{t}-\theta_{k}^{t}\right)\right] \\
& Q_{i}^{t}=Q_{G_{i}}^{t}-Q_{L_{i}}^{t}+Q_{d_{i}}^{t}-Q_{\mathrm{ch}_{i}}^{t} \\
& =\sum_{i=1}^{n} V_{i}^{t} V_{k}^{t}\left[G_{i k} \sin \left(\theta_{i}^{t}-\theta_{k}^{t}\right)-B_{i k} \cos \left(\theta_{i}^{t}-\theta_{k}^{t}\right)\right] .
\end{aligned}
$$

2) ESS energy balance equations: Include energy balance equations for each ESS $i$ in each period $t$, considering charging and discharging efficiencies

$$
B_{i}^{t}=B_{i}^{t-1}+\left(\eta_{\mathrm{ch}_{i}} P_{\mathrm{ch}_{i}}^{t}-P_{d_{i}}^{t} / \eta_{d_{i}}\right) \Delta t .
$$

3) Inequality constraints.

Upper and lower limits for voltage magnitudes

$$
V_{i}^{\min } \leq V_{i}^{t} \leq V_{i}^{\max } .
$$

Bounds on real and reactive generation powers

$$
\begin{aligned}
& P_{G_{i}}^{\min } \leq P_{G_{i}}^{t} \leq P_{G_{i}}^{\max } \\
& Q_{G_{i}}^{\min } \leq Q_{G_{i}}^{t} \leq Q_{G_{i}}^{\max } .
\end{aligned}
$$

Branch current limits

$$
\begin{aligned}
& \left(I_{i j}^{t}\right)^{2} \leq\left(I_{i j}^{\max }\right)^{2} \\
& \left(I_{j i}^{t}\right)^{2} \leq\left(I_{j i}^{\max }\right)^{2} .
\end{aligned}
$$

ESS charging/discharging power bounds

$$
\begin{aligned}
& P_{d_{i}}^{\min } \leq P_{d_{i}}^{t} \leq P_{d_{i}}^{\max } \\
& P_{c h_{i}}^{\min } \leq P_{\mathrm{ch}_{i}}^{t} \leq P_{\mathrm{ch}_{i}}^{\max } \\
& Q_{d_{i}}^{\min } \leq Q_{d_{i}}^{t} \leq Q_{d_{i}}^{\max } \\
& Q_{\mathrm{ch}_{i}}^{\min } \leq Q_{\mathrm{ch}_{i}}^{t} \leq Q_{\mathrm{ch}_{i}}^{\max } .
\end{aligned}
$$

ESS energy limits

$$
B_{i}^{\min } \leq B_{i}^{t} \leq B_{i}^{\max } .
$$

When the ESS is discharged, constraint (10) must be fulfilled. Similarly, when it is charged, constraint (11) must be satisfied.

The aforementioned OPF problem is formulated as a sparse and complete model, hence, the Lagrange multiplier $\lambda p_{i}^{t}$ associated to the real power flow equation at bus $i$ in period $t$ 
represents the variation of the total production cost with respect to the variation of real injected power at the same bus, i.e., it is the locational marginal price (LMP) at bus $i$ in period $t$

$$
\lambda p_{i}^{t}=\mathrm{LMP}_{i}^{t}=-\frac{\partial \mathrm{PC}^{t}}{\partial P_{i}^{t}} .
$$

According to the formulation of the OPF model described previously, $\lambda p_{i}^{t}$ includes the effects of both real losses and congestions.

The aforementioned ac OPF model can be applied for any ESS technology, and in Section III, ESSs of battery technology are employed. This ac OPF formulation has been implemented into a multiperiod OPF model, using MATLAB 2013b software.

\section{B. Assessment of Sensitivity}

From the information provided by the Lagrange multiplier $\lambda p_{i}^{t}$ previously, best candidate buses and worst candidate buses for installing ESSs are identified. Indeed, buses with the highest Lagrange multipliers are selected as the best candidate buses, where any variation of real injected power has greater impact on the production cost than other buses. As a result, if the ESSs are installed at the best candidate buses, their operation will have higher influence on the production cost. In particular, the procedure is described as follows.

1) First, a base case OPF (without ESS installed) is solved. In this way, the Lagrange multiplier $\lambda p_{i}^{t}$ is determined for each bus $i$ at each hour $t$. At this step, constraints on ESSs, including (4) and (10)-(14), are removed from the OPF problem. Next, the following parameter $d f_{i}$ is computed for each bus $i$

$$
d f_{i}=\sum_{t=1}^{T}\left|\lambda p_{i}^{t}\right| .
$$

This parameter is then sorted: The highest values indicate the most suitable buses for the installation of ESSs. The lowest values, on the other hand, indicate the less sensitive candidates. The aforementioned parameter takes into account the effect of the ESSs not only for a specific hour, but considering the whole time horizon.

2) Second, based on the total number of ESSs available, they are connected to the system at the best candidate buses and the OPF problem, with all constraints included, is solved.

In the following section, a set of tests is performed to discuss both the time-shifting and congestion mitigation applications. In each test, production costs, total amount of curtailed wind power and hourly LMP variation are calculated and shown.

\section{CASE Studies And Discussion}

In this section, tests are performed with modified IEEE 14bus and IEEE 118-bus systems. Wind data are taken from real wind records measured at a wind farm in Sicily, Italy. Load data are also relevant to the typical load of a winter day in Italy. Both wind and load data are suitably scaled down to fit the case studies.

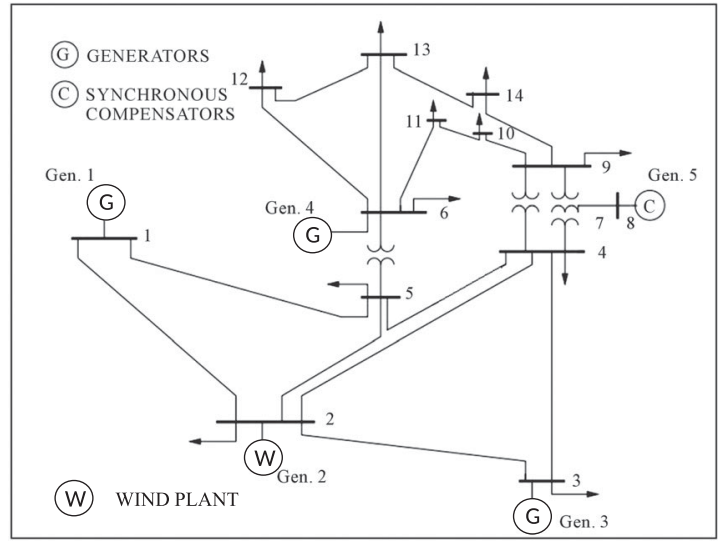

Fig. 1. IEEE 14-bus case study.

TABLE I

PARAMETERS FOR THE BES

\begin{tabular}{lcccc}
\hline \hline$P_{c h}^{\max }[\mathrm{MW}]$ & $P_{d}^{\max }[\mathrm{MW}]$ & $B^{\max }[\mathrm{MW} \cdot \mathrm{h}]$ & $\eta_{\mathrm{ch}}$ & $\eta_{d}$ \\
\hline 30 & 30 & 120 & 0.90 & 0.90 \\
\hline \hline
\end{tabular}

\section{A. IEEE 14-Bus System}

The mathematical model described in Section II is tested on the modified IEEE 14-bus system (see Fig. 1) [26]. This network has four conventional generators (at buses 1, 3, 6, and 8) with total capacity of $832 \mathrm{MW}$, a wind plant (at bus 2) with installed capacity of $250 \mathrm{MW}$ and BESs. BESs are added to support wind generation due to its intermittent behavior to possibly reduce wind curtailment, congestions, and improve the overall economics.

Parameters for the BES are provided in Table I for different tests carried out.

In this system, loads with peak value of $732 \mathrm{MW}$ are supplied from both conventional and wind generation. When the wind is sufficient, it will be the priority source to supply loads and if there is still surplus wind power, BESs will be charged. When wind power is not sufficient, BESs will be discharged to supply loads while respecting all technical constraints. If both wind and BES stored energy are not enough for the loads, conventional generators will be dispatched consequently.

From the OPF formulation described in Section II, the Lagrange multipliers of real power at each bus in each hour are determined. The parameter $d f_{i}$ is then calculated for each bus $i$, including the wind bus and load buses (see Table II).

From this table, the first five buses $(14,10,9,13$, and 7) with highest values of Lagrange multipliers are selected as the best candidate buses to install the BESs. It is worth noticing that the wind bus (bus 2) is among the worst candidate buses.

Next, different cases where different numbers of BES are placed in the system are considered to assess the quality of the sensitivities computed. The tests are categorized as in Table III.

The large BES in cases 7 and 8 is equivalent to two BESs in cases $1-6$ connected to the same bus. 
TABLE II

VALUES OF THE PARAMETER $d f_{i}$ AT EACH BUS

\begin{tabular}{lccc}
\hline \hline $\begin{array}{l}\text { Best Candidate } \\
\text { Bus No. }\end{array}$ & $\begin{array}{c}d f_{i} \\
{[\$ / \mathrm{MW} \cdot \mathrm{h}]}\end{array}$ & $\begin{array}{c}\text { Worst Candidate } \\
\text { Bus No. }\end{array}$ & $\begin{array}{c}d f_{i} \\
{[\$ / \mathrm{MW} \cdot \mathrm{h}]}\end{array}$ \\
\hline 14 & 1739.16 & 11 & 1615.55 \\
10 & 1662.22 & 4 & 1610.09 \\
9 & 1657.42 & 12 & 1605.50 \\
13 & 1633.53 & 5 & 1569.58 \\
7 & 1622.41 & 2 & 1485.79 \\
\hline \hline
\end{tabular}

TABLE III

TESTS FOR THE IEEE 14-BUS SYSTEM

Case $0 \quad$ No BES connected to the network

Case 1 One BES connected to bus 2 (the worst candidate bus)

Case 2 One BES connected to bus 14 (the best candidate bus)

Case 3 Two BESs, one at bus 2, the other at bus 14

Case 4 Two BESs connected to buses 9 and 14 (best candidate buses)

Case 5 Three BESs, one at bus 2, the others at buses 9 and 14

Case 6 Three BESs connected to buses 9,10 and 14 (best candidate buses)

Case 7 One large BES connected to bus 2

Case 8 One large BES connected to bus 14

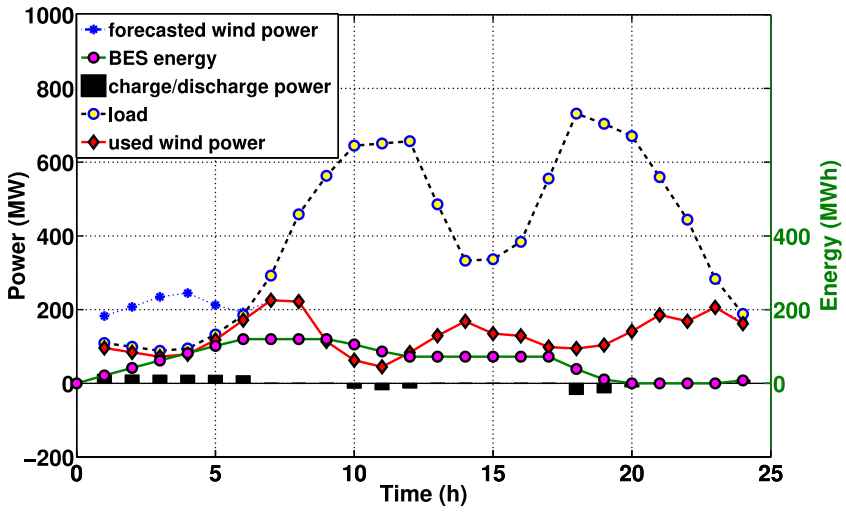

Fig. 2. Operational schedule of the BES in case 2 .

The optimization problem is run for a period of $24 \mathrm{~h}$. Operations of the BES for case 2, with one BES connected to bus 14 (the best candidate bus), are represented in Fig. 2.

As shown in the figure, the BES is charged when wind power exceeds the load, and then, it is discharged when wind power is insufficient to supply the load. At periods when the wind is much higher than the load, after the storage has been charged up to its limits, either power or energy limit, the extra wind is necessarily curtailed.

To understand the operation of the BESs in each case, the resulting production costs, amounts of curtailed wind power, and LMPs of the aforementioned cases will be compared.

1) Production Costs: Production cost is the cost for generating real power by the generating units only (not including generating cost by the storages). Generation costs of all eight cases are shown in Fig. 3. It can be clearly seen from this figure that the case without BESs yields highest generation cost over the other cases. Cases with only one BES connected to the network result in a noticeable reduction of generation cost and this

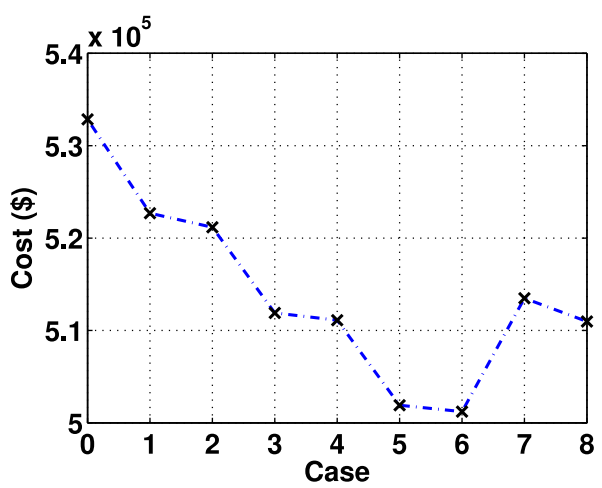

Fig. 3. Production costs of all cases.

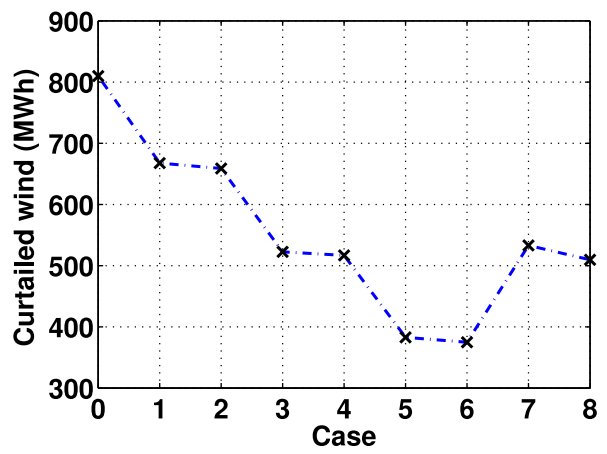

Fig. 4. Amount of curtailed wind of all cases.

reduction is higher in the case when the BES is connected to the best candidate bus (case 1 achieves about $1.9 \%$ cost savings, while case 2 obtains approximately $2.19 \%$ cost savings compared to case 0). In cases 3 and 4, with two BESs, and cases 5 and 6, with three BESs connected to the system, the production cost is further reduced. In general, the selection of the best candidate buses improves the system operation, although the effect due to the total BES capacity looks more significant in this case. Quality of sensitivities computed can be evaluated by comparing cases 1 and 2 and 7 and 8: The comparison shows that the candidate buses for BES installation are actually correctly identified. Also, from the aforementioned analysis, it can be observed that higher capacity of the BES added to the network can significantly improve the overall economics of the system.

2) Curtailed Wind Energy: Wind is curtailed once there is surplus wind but BESs have already reached their capacity limit, either power or energy limit. This curtailment of the wind can be viewed as an undesirable loss of "cost free" and clean energy.

From Fig. 2, the wind is possibly curtailed from hours 1 to 5, in which wind is higher than load. Total amount of the curtailed wind for each case can be seen in Fig. 4. Amounts of curtailed wind energy in all eight cases vary similarly as the generation costs. It is worth noticing that case 2 uses more wind power than case 1 even BES in case 2 is located far from the wind bus. The same occurs when comparing cases 7 and 8 . Hence, it is important to observe that the computed sensitivities correctly take into account wind curtailment also. 


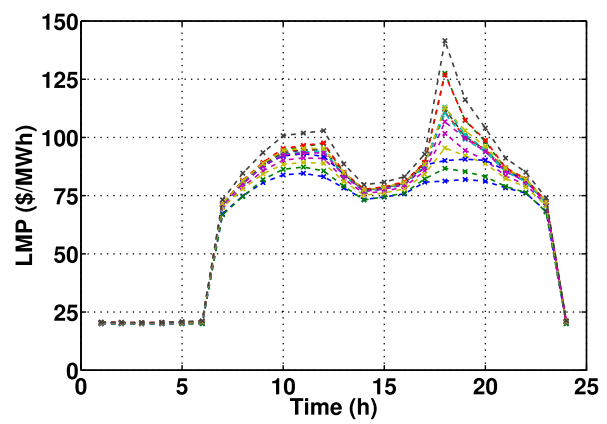

Fig. 5. Hourly LMP variation in case 0 .

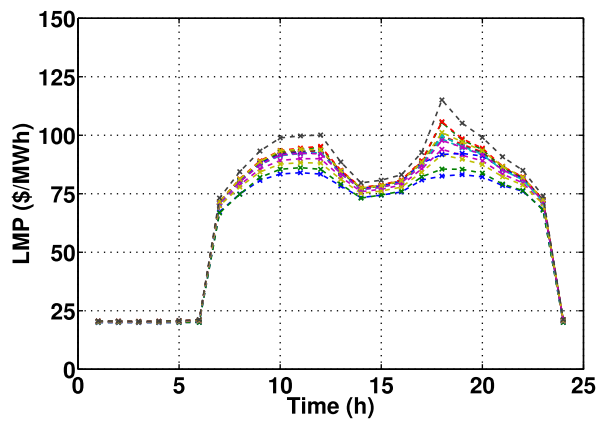

Fig. 6. Hourly LMP variation in case 1 .

3) LMPs: LMP is an important price indicator of unit $\mathrm{MW} \cdot \mathrm{h}$ injection at each node and congestion in the transmission network [27]. It consists of marginal unit cost, congestion cost, and cost due to losses. Castillo and Gayme [25] demonstrated that LMPs play a significant role in driving storage operation at low levels of ESS integration. In this study, we examine how LMPs are changed due to the addition of BESs at different locations.

The hourly LMP variation of all 14 buses in case 0 are presented in Fig. 5. During peak load periods, LMPs also reach peak values while during off-peak hours their values become much lower. This is understandable since at peak load hours, cheap wind power is not sufficient to supply the load and more expensive conventional generators are dispatched instead, which causes an increase in LMPs.

Fig. 6 shows LMPs of case 1, in which a BES is connected to bus 2 (the worst candidate bus). In this case, peak prices are noticeably reduced for the higher peak (hours 18-20), from peak value of about $140 \$ / \mathrm{MW} \cdot \mathrm{h}$ to around $115 \$ / \mathrm{MW} \cdot \mathrm{h}$. The lower peak (during hours 10-12) is also slightly reduced (from peak value of about $105 \$ / \mathrm{MW} \cdot \mathrm{h}-100 \$ / \mathrm{MW} \cdot \mathrm{h}$ ). This indicates that the addition of the BES can provide additional cheap power to loads during peak periods. The reduction in LMP values in this case will affect the cost of supplying load at each bus.

In case 2 (see Fig. 7), both peaks are further reduced. The second peak (hours 18-20) is considerably reduced and becomes almost equal to the first peak (hours 10-12), i.e., about $90 \$ / \mathrm{MW} \cdot \mathrm{h}$. This indicates the addition of BESs at a bus in the best candidate buses has more significant influence on marginal prices than the addition of BESs at a bus in the worst candidate buses, which means the computation of sensitivities is correct. For the remaining cases, similar conclusions can be drawn.

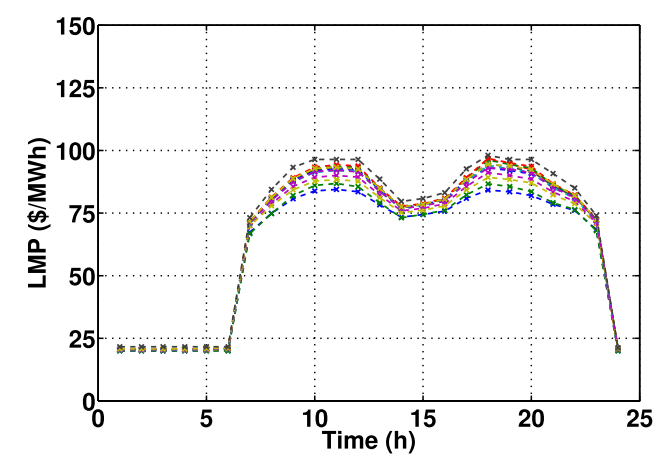

Fig. 7. Hourly LMP variation in case 2.

TABLE IV

SElected Best AND Worst CANDidate Buses

\begin{tabular}{lccc}
\hline $\begin{array}{l}\text { Best Candidate } \\
\text { Bus No. }\end{array}$ & $\begin{array}{c}d f_{i} \\
{[\$ / \mathrm{MW} \cdot \mathrm{h}]}\end{array}$ & $\begin{array}{c}\text { Worst Candidate } \\
\text { Bus No. }\end{array}$ & $\begin{array}{c}d f_{i} \\
{[\$ / \mathrm{MW} \cdot \mathrm{h}]}\end{array}$ \\
\hline 5 & 658.28 & 37 & 581.38 \\
3 & 654.75 & 114 & 581.21 \\
7 & 650.06 & 115 & 581.18 \\
2 & 649.77 & 23 & 576.83 \\
11 & 648.80 & 38 & 575.17 \\
117 & 647.73 & 17 & 574.39 \\
13 & 637.95 & 30 & 552.03 \\
14 & 631.46 & 8 & 491.40 \\
109 & 624.09 & 9 & 486.10 \\
16 & 623.80 & 10 & 480.64 \\
\hline \hline
\end{tabular}

\section{B. IEEE 118-Bus System}

To further investigate the sensitivity of the BES location and size in congestion relieving application in a large network, an extensive set of tests is carried out on the modified IEEE 118bus system [26]. The test system has 56 conventional generating units with a total capacity of $2500 \mathrm{MW}$, two large wind farms connected to buses 8 and 10 with a total installed capacity of 700 MW. Load with peak value of 2189 MW is supplied from both conventional and wind generators. Generation from the wind farms is likely to cause congestion on the way from wind to loads, which might result in wind curtailment. In this case, BESs are installed to charge this otherwise curtailed wind amount for later releasing and allow an efficient utilization of transmission lines. For this test, a congestion is observed during peak periods on lines $8-5$ and $8-30$, from wind farms to loads. Parameters for the BESs are the same as in the previous case study.

The calculated parameter $d f_{i}$, the best candidate, and worst candidate buses for installing BESs are selected as shown in Table IV.

From this table, the best candidate buses include buses 5, 3, $7,2,11,117,13,14,109$, and 16, while the worst candidate buses include buses 37, 114, 115, 23, 38, 17, 30, 8, 9, and 10. It is worth noticing that wind buses ( 8 and 10) are not in the best candidate set as the optimization is carried out from a system point of view. The tests carried out are described in Table V.

1) Production Costs: In Fig. 8, a plot of production costs of the system in all cases is provided. Case 0 yields the highest cost 
TABLE V

TESTS FOR THE IEEE 118-BUS SySTEM

\begin{tabular}{ll}
\hline \hline Case 0 & No BES connected to the network \\
Case 1 & One BES connected to bus 8 (one of the worst candidate buses) \\
Case 2 & One BES connected to bus 5 (best candidate bus) \\
Case 3 & Two BESs, one at bus 8 and the other at bus 5 \\
Case 4 & Two BESs connected to buses 5 and 117 (best candidate buses) \\
Case 5 & Three BESs, one at bus 8 , the others at buses 5 and 117 \\
Case 6 & Three BESs, two at buses 8 and 10, the other at bus 5 \\
Case 7 & Three BESs connected to buses 2, 5, and 117 \\
Case 8 & One large BES system connected to bus 8 \\
Case 9 & One large BES system connected to bus 5 \\
\hline \hline
\end{tabular}

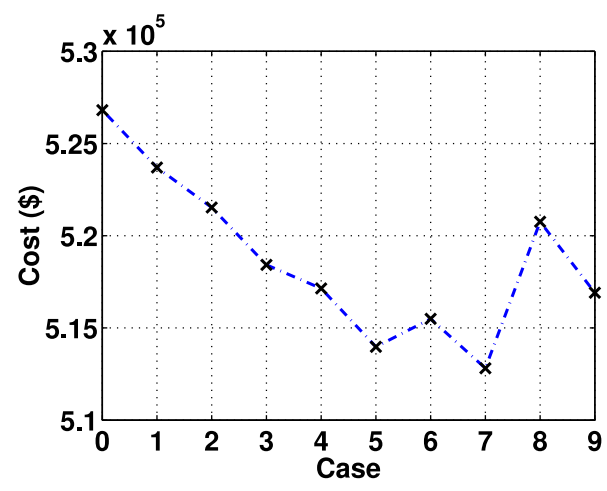

Fig. 8. Production costs of cases 0-9.

compared to the others. From cases 1 to 5, the cost is gradually reduced. This demonstrates that higher numbers of the BES installed return more economical operations of the system. The effectiveness of a good selection of candidate buses by the sensitivity computation is clear by looking at cases 1 and 2: Case 2 results in higher saving as compared to case 1 . Similarly, cases 4 and 7 , where there are more BESs installed at the best candidate buses, also result in lower production costs compared to case 3 , and cases 5 and 6, respectively. Cost savings in case 8 is lower than that in case 3 and case 9. From this analysis, it can be deduced that in such a congested system, storage devices placed at the best candidate buses can provide far more economical operation than those placed at the worst candidate buses. Thus, it is important to figure out the best candidate locations for the planning of storage devices. Moreover, in this case, a large BES connected to a bus in the best candidate buses can operate as efficiently as several BESs distributed among the best candidate buses.

2) Curtailed Wind Energy: Amounts of curtailed wind energy in all cases are represented in Fig. 9. Considering cases 1 and 2, for instance, the conclusion is that the difference in total cost (see Fig. 8) is not due to wind curtailment, like for the aforementioned 14 bus test system, but due to congestions: The optimal placing of BES allows, in this case, to best relieve the congestion due to wind power. In this case, the amount of wind energy to be curtailed is not affected by centralized or decentralized placement of the storage devices.

3) LMPs: Hourly LMP variation of all buses for each case is also shown to discuss the impacts of BES location and size on LMPs. Hourly LMP variation of case 0 is shown in Fig. 10. In

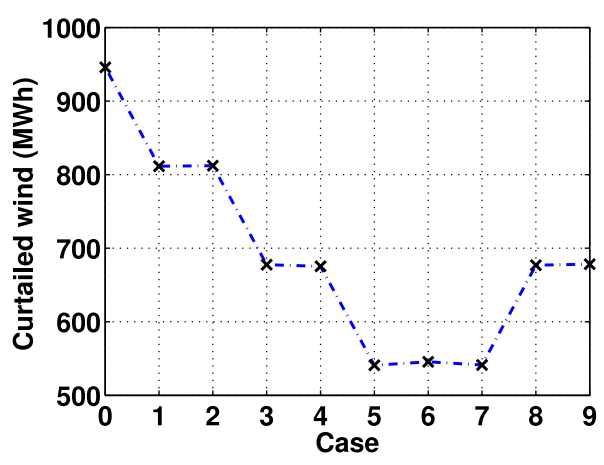

Fig. 9. Amounts of curtailed wind of cases 0 to 9 .

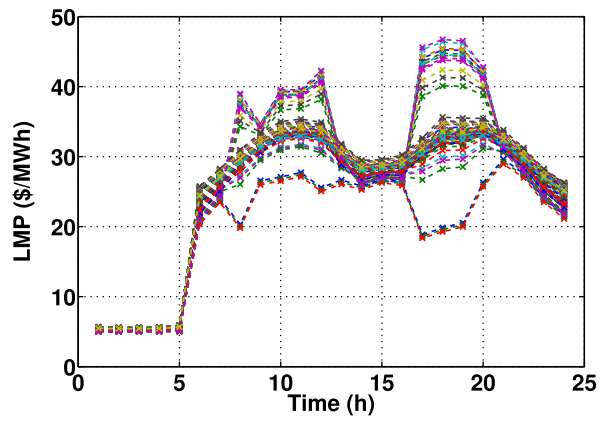

Fig. 10. Hourly LMP variation of case 0 .

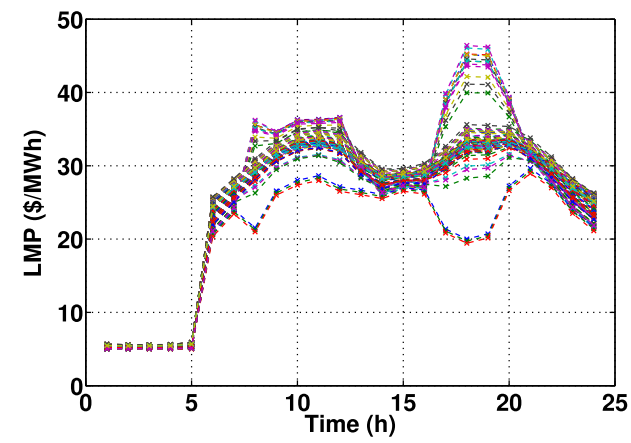

Fig. 11. Hourly LMP variation of case 2 .

this figure, curves with the highest peaks belong to load buses on the receiving side of congested lines and curves with the lowest prices belong to wind buses. During off-peak periods, LMPs are about the same for all buses since there is no congestion.

Hourly LMP variation in case 1, when there is one BES connected to bus 8 (one of the worst candidate buses), is basically the same as in case 0 . In case 2, with one BES added at bus 5 (the best candidate bus), the higher peak prices during the first peak hours are noticeably lowered and those during the second peak hours are also considerably lowered at some periods (hours 17 and 20) as shown in Fig. 11. The peak prices are not reduced at hours 18 and 19 of the second peak period because the limited capacity of the BES is not enough to supply the high load during these hours. For these peak prices to get reduced, higher capacity of the BES is required. In this case, 


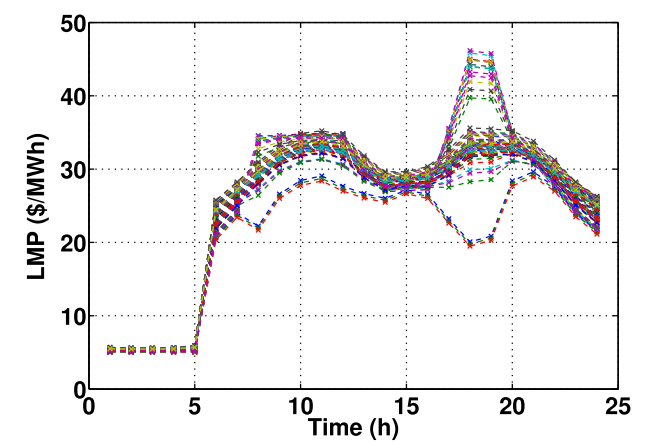

Fig. 12. Hourly LMP variation of case 4 .

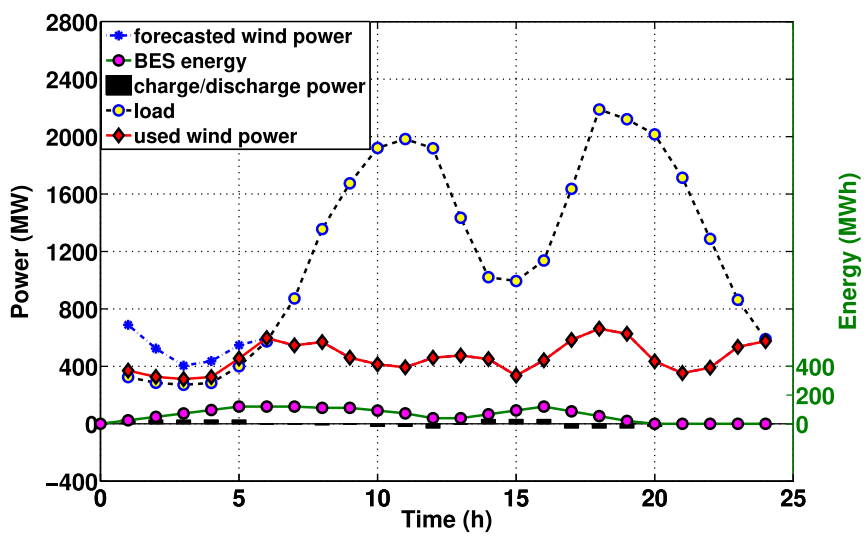

Fig. 13. Operation of the BES in case 2.

to avoid transmission congestion, the BES is charged by wind power during low load periods, when there is no congestion, and then, discharged to supply the cheap energy to loads during congestion hours, hence it can help to reduce the marginal cost during peak hours of these load buses. This operation of the BES has effectively supported wind generation and efficiently makes use of the available transmission capacity.

In case 4 (two BESs connected to the best candidate buses), LMP values of the higher peak curves are further reduced during the first peak periods and during hours 17 and 20 of the second peak (see Fig. 12).

Now, a plot is provided (see Fig. 13) showing the operation of the BES connected to bus 5 (the best candidate bus) to exclusively examine how it shifts wind to avoid transmission constraint.

From this figure, the BES is charged during off-peak periods (hours 1-5 and hours 14-16), which are also periods without transmission congestion, and then, discharged during peak periods (hours 10-12 and hours 17-20) when congestion occurs. Clearly, the BES has thoroughly shifted wind power from wind side to load side to supply loads when wind power cannot be transferred from wind farms to loads due to limited transmission capacity.

Also, in Fig. 14, is a plot of power flows on line 8-5, connecting wind farms and loads. The dotted line with crosses in the plot corresponds to the unconstrained case without BES: the power flow limit (the dotted red line with filled circle) is not enforced by the optimization procedure and this would cause the

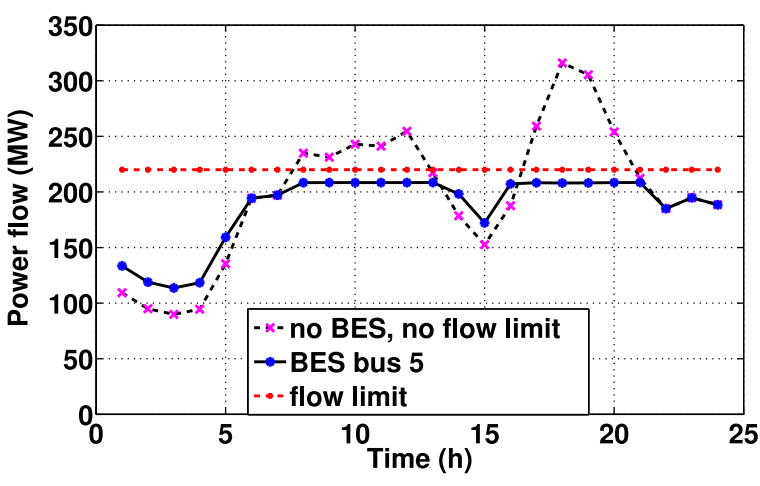

Fig. 14. Power flow on line $8-5$ in case 2.

real-time curtailment of excess wind power. This figure clearly illustrates the alternative path that the BES provides for wind power to alleviate the congestion, i.e., the full blue line with circles. In this way, power flow on line 8-5 during low load hours (hours 1-5 and 14-16) is increased but still lower than the flow limit. Such flow increase is due to the wind power flow used to charge the BES at load bus. This stored energy is released to supply loads during peak periods, when congestion occurs. Consequently, wind power can still be supplied to loads while ensuring the flow limit.

\section{CONCLUSION}

In this paper, the problem of selecting the best location for ESS installation is faced. A multiperiod full ac OPF is used to determine the sensitivities necessary to identify the buses that, in case of installation of ESSs, allow the maximum benefit for power systems from several points of view: the minimum overall cost, the minimum curtailment of wind power (that could also lead to minimum $\mathrm{CO}_{2}$ emissions), the maximum mitigation of congestions, and the maximum benefit, in terms of energy process. The sensitivities are computed as a byproduct of a multiperiod ac OPF, thus taking into account not only a single hour but the overall time horizon and possible time shifts of generated wind power, as well as issues related to congestions mitigation and reduction of losses. The proposed methodology has been tested on two test systems using realistic data, and the sensitivities have been assessed, showing a very informative content. Moreover, the system benefits have been proved with reference to LMPs, used here as an index of the social benefit. The results demonstrate that the method can be easily applied to large systems and to many different scenarios to take into account the variability of both wind power and load.

\section{REFERENCES}

[1] EPRI-DOE, Handbook of Energy Storage for Transmission and Distribution Applications, Electric Power Research Institute, Palo Alto, CA, USA, 2004.

[2] R. B. Schainker, "Executive overview: Energy storage options for a sustainable energy future," in Proc. IEEE Power Eng. Soc. Gen. Meeting, vol. 2, Jun. 2004, pp. 2309-2314.

[3] C. Abbey and G. Joos, "Supercapacitor energy storage for wind energy applications," IEEE Trans. Ind. Appl., vol. 43, no. 3, pp. 769-776, May 2007. 
[4] P. Mercier, R. Cherkaoui, and A. Oudalov, "Optimizing a battery energy storage system for frequency control application in an isolated power system," IEEE Trans. Power Syst., vol. 24, no. 3, pp. 1469-1477, Aug. 2009.

[5] R. Rajagopal, E. Bitar, P. Varaiya, and F. Wu, "Risk-limiting dispatch for integrating renewable power," Int. J. Elect. Power Energy Syst., vol. 44, no. 1 , pp. 615-628, 2013.

[6] E. Sjodin, D. Gayme, and U. Topcu, "Risk-mitigated optimal power flow for wind powered grids," in Proc. Amer. Control Conf., Jun. 2012, pp. 4431-4437.

[7] C. Brivio, M. Mandelli, and M. Merlo, "Battery energy storage system for primary control reserve and energy arbitrage," Sustainable Energy, Grids Netw., vol. 6, pp. 152-165, Jun. 2016.

[8] S. Gill, G. Ault, and I. Kockar, "The optimal operation of energy storage in a wind power curtailment scheme," in Proc. Power Energy Soc. Gen. Meeting, Jul. 2012, pp. 1-8.

[9] H. Daneshi and A. Srivastava, "Impact of battery energy storage on power system with high wind penetration," in Proc. IEEE PES Transmiss. Distrib. Conf. Expo., May 2012, pp. 1-8.

[10] H. Akhavan-Hejazi and H. Mohsenian-Rad, "Optimal operation of independent storage systems in energy and reserve markets with high wind penetration," IEEE Trans. Smart Grid, vol. 5, no. 2, pp. 1088-1097, Mar. 2014.

[11] W. Hu, Z. Chen, and B. Bak-Jensen, "Optimal operation strategy of battery energy storage system to real-time electricity price in Denmark," in Proc. Power Energy Soc. Gen. Meeting, Jul. 2010, pp. 1-7.

[12] J. Perez-Diaz, A. Perea, and J. Wilhelmi, "Optimal short-term operation and sizing of pumped-storage power plants in systems with high penetration of wind energy," in Proc. 7th Int. Conf. Eur. Energy Market, Jun. 2010, pp. 1-6.

[13] Y. Atwa and E. El-Saadany, "Optimal allocation of ESS in distribution systems with a high penetration of wind energy," IEEE Trans. Power Syst., vol. 25, no. 4, pp. 1815-1822, Nov. 2010.

[14] J. Song, T. Brekken, E. Cotilla-Sanchez, A. von Jouanne, and J. Davidson, "Optimal placement of energy storage and demand response in the pacific northwest," in Proc. IEEE Power Energy Soc. Gen. Meeting, Jul. 2013, pp. 1-5.

[15] H. Oh, "Optimal planning to include storage devices in power systems," IEEE Trans. Power Syst., vol. 26, no. 3, pp. 1118-1128, Aug. 2011.

[16] M. Ghofrani, A. Arabali, M. Etezadi-Amoli, and M. Fadali, "A framework for optimal placement of energy storage units within a power system with high wind penetration," IEEE Trans. Sustainable Energy, vol. 4, no. 2, pp. 434-442, Apr. 2013.

[17] M. Ghofrani, A. Arabali, M. Etezadi-Amoli, and M. S. Fadali, "Energy storage application for performance enhancement of wind integration," IEEE Trans. Power Syst., vol. 28, no. 4, pp. 4803-4811, Nov. 2013.

[18] S. B. K. Dvijotham and M. Chertkov, "Operations-based planning for placement and sizing of energy storage in a grid with a high penetration of renewables," Los Alamos Nat. Lab., Tech. Rep. arXiv:1107.1382 [math.OC], 2011.

[19] S. Bose, D. F. Gayme, U. Topcu, and K. M. Chandy, "Optimal placement of energy storage in the grid," in Proc. IEEE 51st Annu. Conf. Decision Control, 2012, pp. 5605-5612.

[20] S. Wogrin and D. F. Gayme, "Optimizing storage siting, sizing, and technology portfolios in transmission-constrained networks," IEEE Trans. Power Syst., vol. 30, no. 6, pp. 3304-3313, Nov. 2015.

[21] P. Xiong and C. Singh, "Optimal planning of storage in power systems integrated with wind power generation," IEEE Trans. Sustain. Energy, vol. 7, no. 1, pp. 232-240, Jan. 2016.

[22] N. Nguyen, D. Le, G. Moshi, C. Bovo, and A. Berizzi, "Sensitivity analysis on locations of energy storage in power systems with wind integration," in Proc. IEEE 15th Int. Conf. Environ. Elect. Eng., Jun. 2015, pp. 1115-1119.

[23] N. Nguyen, D. Le, C. Bovo, and A. Berizzi, "Optimal power flow with energy storage systems: Single-period model versus multi-period model," in Proc. IEEE Eindhoven PowerTech, 2015, pp. 1-6.

[24] B. Klöckl, G. Papaefthymiou, and P. Pinson, "Probabilistic tools for planning and operating power systems with distributed energy storage," $E \& I$ Elektrotechnik und Informationstechnik, vol. 125, no. 12, pp. 460-465, 2008.

[25] A. Castillo and D. Gayme, "Profit maximizing storage allocation in power grids," in Proc. IEEE 52nd Annu. Conf. Decision Control, Dec. 2013, pp. 429-435.

[26] University of Washington. (1993). Power system test case archive. [Online]. Available: https://www.ee.washington.edu/research/pstca

[27] A. Kumar and W. Gao, "Optimal distributed generation location using mixed integer non-linear programming in hybrid electricity markets," IET Gener., Transmiss. Distrib., vol. 4, no. 2, pp. 281-298, Feb. 2010. 